\title{
Alcohol-induced autophagy via upregulation of PIASy promotes HCV replication in human hepatoma cells
}

\author{
Meihua Ran ${ }^{1,2}$, Hui Chen 1,2, Bingyu Liang ${ }^{1,3}$, Weibo Liao ${ }^{1,2}$, Junjun Jiang ${ }^{1,3}$, Jiegang Huang ${ }^{1,3}$, Chuanyi Ning ${ }^{1,3}$,

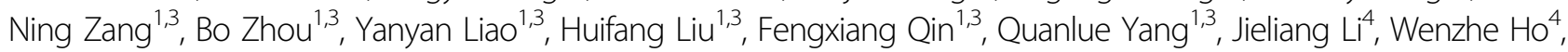 \\ Hao Liang ${ }^{1,3}$ and Li Ye (1) ${ }^{1,3}$
}

\begin{abstract}
Both alcohol and hepatitis C virus (HCV) infection could induce cellular autophagy in liver cells, which is considered to be essential for productive HCV replication. However, whether alcohol-induced autophagy is involved in the pathogenesis of HCV infection is still poorly understood. Alcohol treatment could induce autophagy in Huh7 cells (a hepatoma cell line that supports HCV JFH-1 replication), evidenced by the increase of LC3B-II levels, the conversion of LC3B-I to LC3B-II, and the formation of GFP-LC3 puncta as well as the decrease of p62 level in alcohol-treated cells compared with control cells. Alcohol treatment also significantly increased PIASy (a member of the PIAS family) expression, which can act as a SUMO (small ubiquitin-like modifier protein) E3 ligase to regulate a broader range of cellular processes including autophagy. Overexpression or the silencing expression of PIASy in alcohol-treated Huh7 cells could increase or decrease autophagic activation caused by alcohol treatment, respectively, and thus affect HCV replication correspondingly. In the absence of alcohol, overexpression or silencing expression of PIASy increase or decrease the level of cellular autophagy, judged by the changes of LC3B-II and p62 levels in the presence or absence of chloroquine (CQ), a lysosome inhibitor. More importantly, in the presence of 3-methyladenine (3-MA), an inhibitor in the early stage of autophagy, the effects of overexpression or silencing expression of PIASy on HCV replication were largely blocked. Furthermore, PIASy could selectively drive the accumulation of SUMO1-conjugated proteins, along with upregulation of the expression of several important autophagy factors, including ATG7 and ATG5-ATG12. In conclusion, alcohol promotes HCV replication through activation of autophagy in Huh7 cells, which partly attributes to its induction of PIASy expression. PIASy-enhanced accumulation of SUMO1-conjugated proteins may contribute to its inducing effect of autophagy. Our findings provide a novel mechanism for the action of alcohol-promoting HCV replication in the context of cellular autophagy.
\end{abstract}

Correspondence: Hao Liang (lianghao@gxmu.edu.cn)

Li Ye (yeli@gxmu.edu.cn)

'Guangxi Key Laboratory of AIDS Prevention and Treatment \& Guangxi Universities Key Laboratory of Prevention and Control of Highly Prevalent Disease, School of Public Health, Guangxi Medical University, Nanning 530021 Guangxi, China

${ }^{2}$ Geriatrics Digestion Department of Internal Medicine, The First Affiliated Hospital of Guangxi Medical University, Nanning 530021 Guangxi, China Full list of author information is available at the end of the article. These authors contributed equally: Meihua Ran, Hui Chen and Bingyu Liang Edited by B. Zhivotovsky

\section{Introduction}

Hepatitis $\mathrm{C}$ virus (HCV) infection and alcohol abuse represent the two main causes of chronic liver disease worldwide $^{1,2}$. Currently, it is estimated that approximately 71.1 million individuals globally are living with $\mathrm{HCV}$ infection $^{3}$, and chronic HCV infection may lead to cirrhosis and hepatocellular carcinoma (HCC $)^{4}$. Alcoholic liver disease is a direct consequence of chronic alcohol

\section{(c) The Author(s) 2018}

(c) (i) Open Access This article is licensed under a Creative Commons Attribution 4.0 International License, which permits use, sharing, adaptation, distribution and reproduction c. in any medium or format, as long as you give appropriate credit to the original author(s) and the source, provide a link to the Creative Commons license, and indicate if changes were made. The images or other third party material in this article are included in the article's Creative Commons license, unless indicated otherwise in a credit line to the material. If material is not included in the article's Creative Commons license and your intended use is not permitted by statutory regulation or exceeds the permitted use, you will need to obtain permission directly from the copyright holder. To view a copy of this license, visit http://creativecommons.org/licenses/by/4.0/. 
consumption and is recognized as an important health problem worldwide. Chronic or acute alcohol abuse often leads to liver injury associated with alcoholic hepatitis, liver fibrosis, cirrhosis, and liver cancer ${ }^{5}$. Previous studies have indicated that $\mathrm{HCV}$ infection and alcoholism coexist in a large number of people. Alcoholic individuals have high seroprevalence of $\mathrm{HCV}$ infection ${ }^{1}$, and among patients with chronic $\mathrm{HCV}$ infection, heavy alcohol consumption is rather common ${ }^{6,7}$. HCV and alcohol most likely act synergistically to accelerate the development and progression of liver disease ${ }^{5}$.

The role of alcohol in promoting HCV-related liver diseases has been suggested in a number of clinical investigations. Mechanism research has revealed that alcohol and HCV may synergistically accelerate the development of liver diseases by enhancement of $\mathrm{HCV}$ replication, suppression of innate immunity ${ }^{8,9}$, increased oxidative stress ${ }^{10}$, generation of reactive oxygen species (ROS), iron accumulation, and steatosis induction ${ }^{2,11}$. These findings also imply that the interactions between alcohol and HCV are very complex and need to be further illustrated. Although the introduction of direct-acting antiviral (DAA) therapies for treatment of $\mathrm{HCV}$ infection has dramatically improved treatment responses and represents a milestone in the HCV treatment landscape, better understanding of the underlying mechanisms responsible for the alcohol effect on HCV infection/ replication would provide new insights into their interaction, as well as information for clinical treatment and management of alcoholic patients with chronic HCV infection, which yet does not have standard guidelines for whether or how long alcohol abuse is abstinent before beginning the HCV treatment, even in the DAA era ${ }^{12}$.

Autophagy is predominantly a protective mechanism, acting as a cleanser to remove damaged organelles and cytosolic components ${ }^{13}$. However, recent studies have highlighted the close interplay of autophagy and $\mathrm{HCV}$. HCV has evolved to utilize autophagy to complete its own replication, and autophagy machinery plays an important role in $\mathrm{HCV}$ pathogenesis ${ }^{14,15}$. The autophagy-related proteins, including Beclin 1, LC3, Atg4B, Atg5, Atg7, and Atg12, have been identified to be proviral factors that are important for productive $\mathrm{HCV}$ replication ${ }^{16-20}$.On the other hand, $\mathrm{HCV}$ has the ability to induce autophagy to enhance its replication, HCV can induce the accumulation of autophagosomes, and use autophagosomal membranes as the site for its RNA replication ${ }^{20,21}$. Enhancement of cellular autophagy, by either $\mathrm{HCV}$ infection itself or other non-HCV factors, could increase the production of $\mathrm{HCV}$ viral particles and favor $\mathrm{HCV}$ propagation $^{18,22}$.

Autophagy also plays a pivotal role in the pathogenesis of alcohol-related liver disease ${ }^{23}$. A number of recent reports have shown that alcohol exposure has a significant effect on hepatic autophagy, and most of them support that alcohol can activate hepatic autophagy in vivo, in cultured primary hepatocytes, and in mice models ${ }^{24-28}$, except that a few studies reported impairment of liver autophagy in chronic alcohol-administered mice ${ }^{29}$. Our preliminary data showed that alcohol treatment could induce cellular autophagy in Huh 7 cells, a hepatoma cell line that supports the full cycle of HCV JFH-1 infection/ replication ${ }^{30}$. Although the precise mechanism by which alcohol consumption affects hepatic autophagy remains unclear, alcohol definitely has an impact on autophagy in liver cells. In this case, it is interesting to illuminate whether the alcohol-induced (or alcohol-inhibited) autophagy plays a role in HCV replication. In this study, we examined whether alcohol enhances HCV infection/ replication via regulation of cellular autophagy in human hepatoma Huh7 cells. We also explored the mechanism(s) at cellular and molecular levels involved in alcohol action on $\mathrm{HCV}$ replication in the context of autophagy.

\section{Materials and methods \\ Reagents}

Alcohol was purchased from Kelong Bio Inc. (Chengdu, China). Chloroquine (CQ), 3-methyladenine (3-MA), anti-P62/SQSTM1 antibody, and secondary antibodies for western blot (horseradish peroxidase-conjugated goatanti-rabbit IgG, and goat-anti-mouse IgG) were purchased from Sigma-Aldrich China (Shanghai, China). Anti- $\beta$-actin, anti-ATG7, anti-ATG3 antibodies were purchased from Abcam Trading China (Shanghai, China). Anti-HCV core, anti-ATG5-ATG12, and Lipofectamine3000 were purchased from Thermo Fisher Scientific China (Shanghai, China). Anti-PIASy, anti-LC3B, antiBeclin 1, anti-SUMO1, and anti-SUMO2 were purchased from Cell Signaling Technology China (Shanghai, China), and anti-CYP2E1 and anti-ADH were purchased from Novus Biologicals China (Shanghai, China).

\section{Cell culture, HCV JFH-1 infection, and plasmid/shRNA transfection}

The human hepatoma cell line Huh7 and HCV JFH-1 virus were kindly provided by Dr. Wenzhe Ho (Temple University, Philadelphia, USA). Huh7 cells were cultured in high-glucose Dulbecco's modified Eagle's medium (DMEM), supplemented with $10 \%$ fetal bovine serum (FBS), $100 \mathrm{nM}$ nonessential amino acids (NEAA), $100 \mathrm{U} /$ $\mathrm{mL}$ penicillin, and $100 \mu \mathrm{g} / \mathrm{mL}$ streptomycin. The cells were cultured at $37^{\circ} \mathrm{C}$ in a humidified incubator with an atmosphere of $5 \% \mathrm{CO}_{2}$. Infectious $\mathrm{HCV} \mathrm{JFH-1}$ was generated by transfection of in vitro- transcribed genomic JFH-1 RNA to Huh7 cells as previously described ${ }^{30}$. Infection of Huh7 cells with HCV JFH-1 was carried out at a multiplicity of infection (MOI) of 0.1. PIASy overexpression or silencing expression was carried out by 
transfection of plasmid containing PIASy (pCMV-mycPIASy) or shRNA against PIASy, respectively. shRNAPIASy and control-scrambled shRNA (the sequence is not available from the supplier) were synthesized from Qiagen, China (Shanghai, China). Transfection of plasmids or shRNA to Huh7 cells was carried out using Lipofectamine 3000 from Thermo Fisher Scientific China (Shanghai, China).

\section{Alcohol treatment}

HCV-infected or uninfected Huh7 cells in 24-well or 12-well plates were incubated with or without alcohol (20-80 mM) for up to $144 \mathrm{~h}$. The alcohol concentrations choice for the study was based on our previous studies ${ }^{9,31}$.

\section{Quantitative real-time RT-PCR (RT-PCR)}

Total cellular RNA was extracted from Huh7 cells using TaKaRa MiniBEST and Universal RNA Extraction Kits from Takara Company (Dalian, China). The RNA was then reversely transcribed to cDNA using the reverse transcription kit (Takara, Dalian, China), following the manufacturer's instruction. RT-PCR was performed using SYBR Green PCR Master Mix (Takara, Dalian, China) and a StepOne Plus real-time PCR system (Life Technologies). The special oligonucleotide primers used in this study were listed in Supplementary Table 1.

\section{Western blot}

The cell lysates were prepared using radioimmunoprecipitation assay (RIPA) buffer (MultiSciences Biotech, Hangzhou, China) plus $1 \%$ protease inhibitor cocktail (Asvio Technology, Guangzhou, China). Protein concentrations were determined by DC protein assay. Western blot assay was carried out as previously described $^{32}$. The primary antibodies used in this study were as follows: anti- $\beta$-actin (1:2000), anti-PIASy (1:1000), antiHCV core (1:3000), anti-LC3B (1:2000), anti-p62 (1:2000), anti-Atg7 (1:1000), anti-Atg5- Atg12 (1:1000), antiSUMO1 (1:1000), anti-SUMO2/3 (1:1000), anti-ADH1 (1:1000), and anti-CYP2E1 (1:1000). The secondary antibodies were horseradish peroxidase-conjugated goat-antirabbit $\operatorname{IgG}(1: 10,000)$ or goat-anti-mouse $\operatorname{IgG}(1: 5000)$, respectively. The immunoreactive bands were visualized by SuperSignal West Pico chemiluminescence substrate (Thermofisher, USA). The band densities were measured by Image J software (National Institutes of Health, Bethesda, MD, USA). The values were normalized to $\beta$-actin.

\section{Autophagy analysis by fluorescence microscope}

The Huh7 cells were transfected with lentivirusmediated transient green fluorescent protein (GFP)-LC3 to generate GFP-LC3-expressing cells. The lentiviral vector containing GFP-LC3 fusion gene and lentivirus were purchased from Genechem Company (Shanghai,
China). Autophagy was assessed using GFP-LC3 redistribution in cells that was detected by an inverted fluorescence microscope (Nikon Ti-s). The number of GFPLC3-positive dots per cell was determined in three independent experiments.

\section{Statistical analysis}

Data were expressed as mean \pm standard deviation (SD). All assays were repeated at least three times, and each experiment was performed at least in triplicate. We determined the statistical significance between two groups by Student's $t$ test and among multiple groups by one-way analysis of variance (ANOVA). The $p$ value < 0.05 was considered significant.

\section{Results}

\section{Alcohol treatment promotes HCV JFH-1 replication in Huh7 cells}

We first verified whether alcohol promotes $\mathrm{HCV}$ replication in Huh7 cells. HCV-JFH-1-infected Huh7 cells, at day 3 post infection, were treated with alcohol at different concentrations for $96 \mathrm{~h}$ (Fig. 1a, b) or at $80 \mathrm{mM}$ for different times (Fig. 1c, d). The alcohol concentrations and treatment time selected for the study were based on our previous studies ${ }^{9,31}$, which indicated that alcohol at the concentration of $100 \mathrm{mM}$ or lower had no cytotoxic effect on Huh7 cells. As shown in Fig. 1, when HCV JFH1-infected Huh7 cells were treated with alcohol, the HCV RNA level was significantly increased in a dose-dependent and time-dependent manner (Fig. 1a, c), which was also confirmed at $\mathrm{HCV}$ core protein level by western blot assay (Fig. 1b, d). Therefore, we concluded that alcohol could promote HCV JFH-1 replication in Huh7 cells. In addition, several studies indicated that the effects of alcohol on HCV replication in Huh7 or Huh7-derived cells (Huh7.5 and Huh7.5.1) are involved in alcohol metabolism, and thus the expression of the two main alcohol-metabolizing enzymes, alcohol dehydrogenase (ADH) and cytochrome P450-2E1 (CYP2E1) in Huh7 cells is important for the action of alcohol on HCV. We used western blot to detect the expression of these two enzymes in Huh7 cells and found that, although Huh7 cells express low levels of $\mathrm{ADH}$ and CYP2E1, when the cells were treated with alcohol, the expression of these two enzymes was induced to relatively high levels (Supplementary Fig. 1).

\section{Alcohol treatment induces cellular autophagy in Huh7 cells}

To examine whether alcohol affects cellular autophagy in Huh7 cells, we first investigated whether alcohol modulates the expression of two markers of autophagy, microtubule-associated protein 1 light chain 3 (LC3) and p62. LC3, a cytosolic ubiquitin-like protein, has two forms, LC3-I and LC3-II. During autophagy activation, the soluble form of LC3 (LC3-I) is converted into a 
A

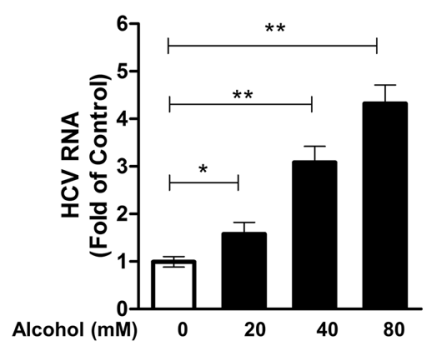

C

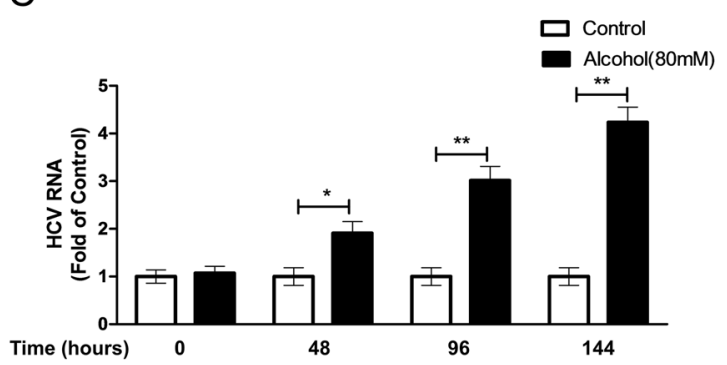

B
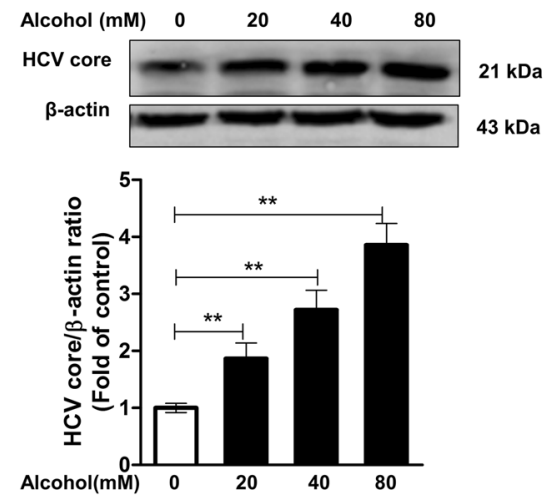

D

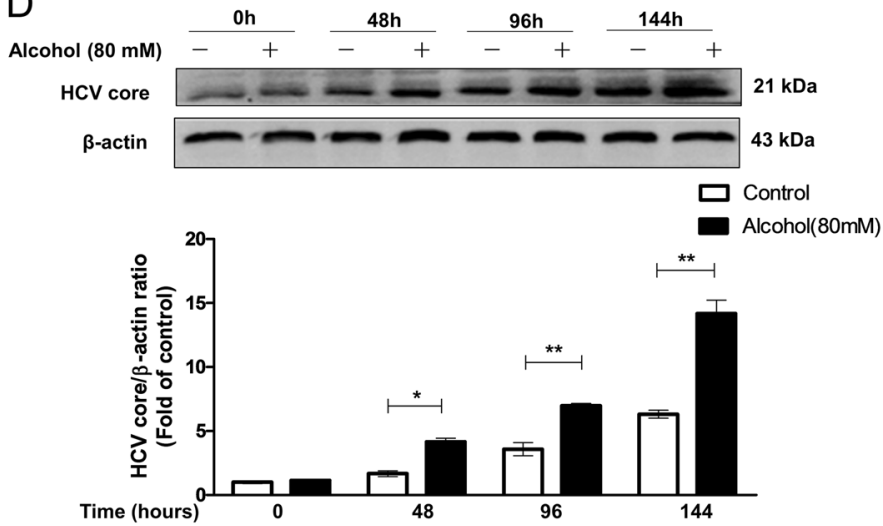

Fig. 1 Alcohol enhances HCV replication in Huh7 cells. Huh7 cells were infected with HCV JFH-1. At day 3 post infection, the cells were treated with or without alcohol under different conditions. $\mathbf{a}, \mathbf{b}$ The dose effect of alcohol on HCV replication. The cellular RNA and proteins were extracted for RT-PCR (a) and western blot analyses (b). a The levels of intracellular HCV RNA, with normalization to the corresponding GAPDH mRNA level, are expressed as the fold of control (without alcohol treatment, which was defined as 1). b A representative western blot image shows HCV core protein expression in alcohol-treated or untreated control cells. The densitometric intensities of the HCV core and $\beta$-actin bands were quantified by Image $\mathrm{J}$ software. The relative HCV core/ $\beta$-actin ratios were calculated and shown as the fold of control (without alcohol treatment, which was defined as 1). c, $\mathbf{d}$ The time-course effect of alcohol on HCV replication. HCV JFH-1-infected Huh7 cells were treated with or without alcohol ( $80 \mathrm{mM})$ for different times $(0,48,96$, and $144 \mathrm{~h})$. The cellular RNA and proteins were extracted for real-time RT-PCR (c) and western blot assay (d). c The levels of intracellular HCV RNA, with normalization to the corresponding GAPDH mRNA level, are expressed as the fold of control (without alcohol treatment, which was defined as 1 at different time points, respectively). $\mathbf{d}$ A representative western blot image shows HCV core protein expression in alcoholtreated or untreated control cells. The relative HCV core/ $\beta$-actin ratios were calculated and shown as the fold of control (without alcohol treatment, which was defined as 1 at different time points, respectively). The data are the mean \pm SD of the results of three independent experiments. ${ }^{*} p<0.05$, ${ }^{* *} p<0.01$

lipidated form (LC3-II), which is associated with autophagosomal membranes. p62, additionally a measure of autophagic flux, is a cargo receptor for autophagic degradation of ubiquitinated targets that binds directly to LC3-II, and is exclusively degraded during autophagy. The decreased levels of p62 could be observed when autophagy is activated. Thus, the relative levels of LC3B-II and p62 were used as the markers of autophagy and were always first analyzed in autophagy. In this study, at the mRNA level, no significant difference $(p>0.05)$ was observed in LC3 or p62 expression between alcoholtreated cells and alcohol-untreated cells (Fig. 2a). At protein level, however, significantly increased levels of LC3B-II protein (LC3B-II/ $\beta$-actin) and conversion of
LC3B-I into LC3B-II (LC3B-II/LC3B-I) were observed in alcohol-treated cells compared with control cells, and the increase was in a dose- and time-dependent manner (Fig. 2b, c). Meanwhile, the levels of p62 (p62/ $\beta$-actin) decreased along with the increasing levels of LC3B-II protein, either in a dose- or time-dependent manner (Fig. 2b, c). Huh7 cells expressing GFP-LC3 were also used to examine the effect of alcohol on the autophagic activity that was indicated by the numbers of GFP-LC3 dots (Fig. 2d). A significant impact of alcohol on GFP-LC3 dots was observed. The numbers of GFP-LC3 dots in alcoholtreated cells were significantly higher than that in control cells, and the effect was obvious in a dose-dependent manner (Fig. 2d). 


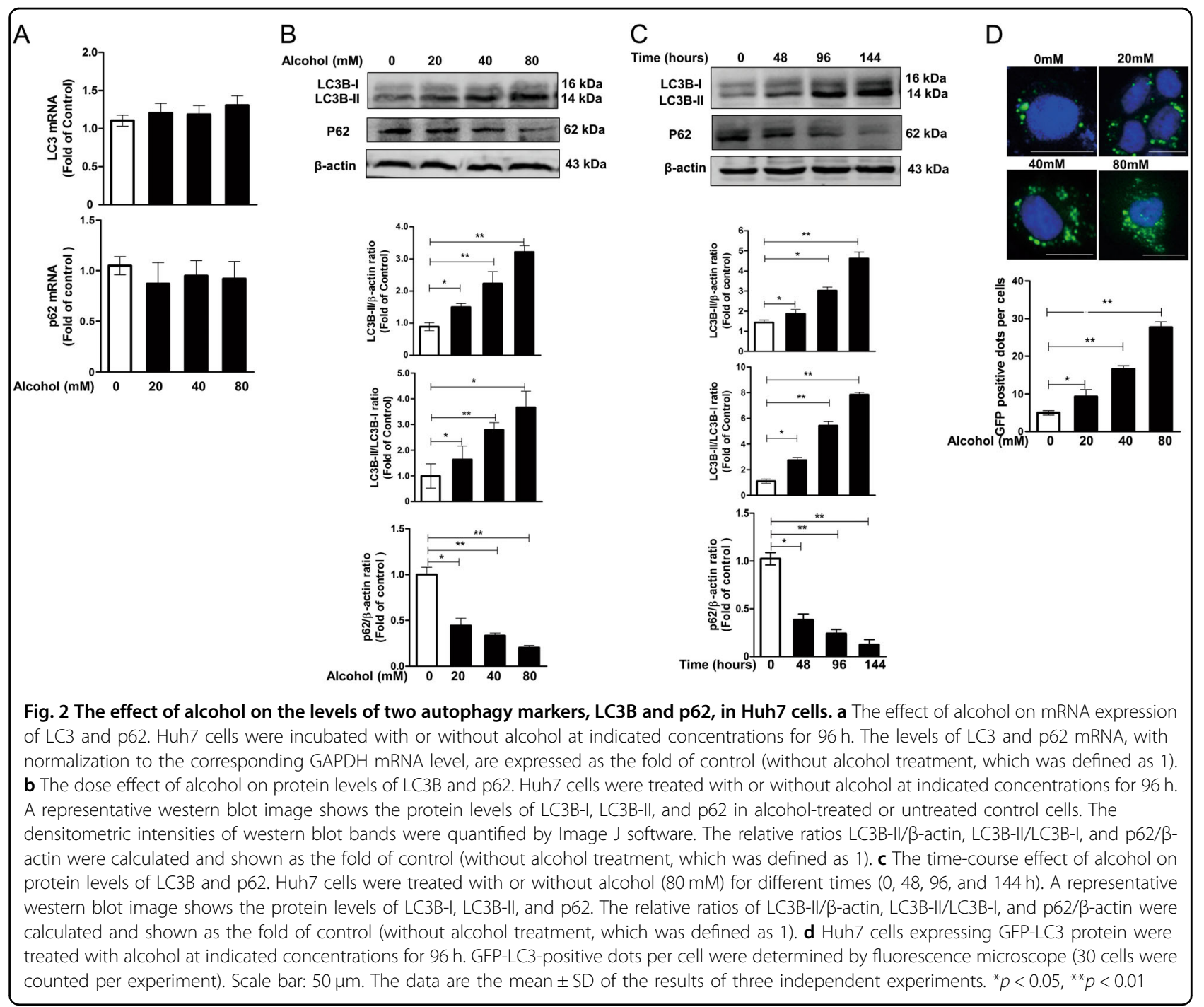

Although the decrease in p62 level does reflect the induction of autophagy, the increase in LC3B-II level could be caused by either induction of autophagosome formation or inhibition of degradation in lysosomes. An autophagosome-lysosome fusion inhibitor, CQ was also used to further clarify whether the increase of LC3B-II levels caused by alcohol was due to the increased autophagosome formation or the decreased autophagosome degradation. The data revealed that the combined use of alcohol and CQ could lead to higher induced LC3B-II level compared with alcohol alone or $C Q$ alone (Fig. 3a-c). However, the CQ treatment had little effect on p62 level because it inhibits the degradation function of lysosomes, and CQ co-treatment could also prevent the degradation of p62 caused by alcohol treatment, which was shown in the alcohol-alone group (Fig. 3a, b). The above results indicated that alcohol could induce cellular autophagy flux in Huh7 cells (Figs. 2 and 3).

\section{Alcohol induces PIASy expression in Huh7 cells}

We then investigated whether alcohol affects the expression of the PIAS family, including PIAS1, PIASx (a, b), PIAS3, and PIASy. At the mRNA level, alcohol treatment significantly upregulated PISAy expression in a dose-dependent and time-dependent manner (Fig. 4a, c). However, no significant effect $(p>0.05)$ was observed in the expression of other PIASs (PIAS1, PIASx(a.b), and PIAS3) (Fig. 4a). Western blot assay confirmed that alcohol could induce PIASy expression in Huh7 cells in a dose-dependent and time-dependent manner (Fig. 4b, d).

\section{Alcohol-induced PIASy is involved in alcohol-enhanced $\mathrm{HCV}$ replication and alcohol-induced autophagy}

To investigate whether alcohol-induced PIASy plays a role in alcohol-enhanced HCV replication and alcoholinduced autophagy, overexpression (pCMV-myc-PIASy) or silencing expression (shRNA-PIASy) of PIASy 


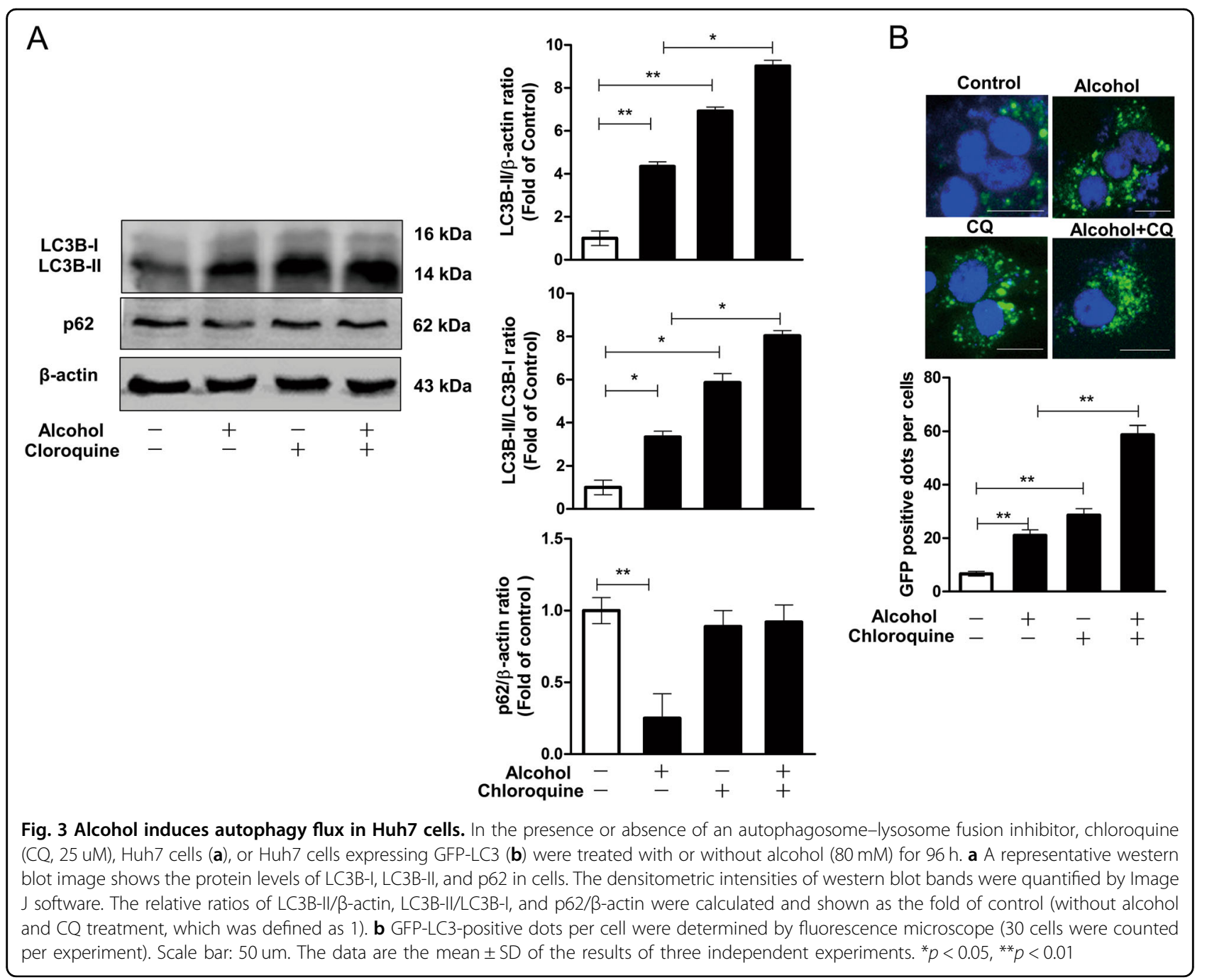

experiments were performed. Overexpression of PIASy could promote $\mathrm{HCV}$ replication and further enhance the promoting effect of alcohol on $\mathrm{HCV}$ replication, evidenced by increased HCV core protein level in PIASytransfected cells compared with control cells (Fig. 5a). Silencing expression of PIASy, on the contrary, inhibited $\mathrm{HCV}$ replication and weakened the alcohol-enhanced HCV replication (Fig. 5c). Similarly, overexpression or silencing expression of PIASy could enhance or weaken alcohol-induced autophagy, respectively, evidenced by the increased levels of LC3B-II (LC3B-II/ $\beta$-actin and LC3BII/LC3B-I) and decreased levels of p62 (p62/ $\beta$-actin) in overexpression groups and opposite changes in silencing groups (Fig. 5b, d).

The effects of PIASy on the autophagy flux in Huh7 cells

Since alcohol-induced PIASy had a significant effect on cellular autophagy, next, we examined whether PIASy itself affects autophagy via overexpression or silencing expression of PIASy in Huh7 cells, in the presence or absence of CQ. In the absence of $\mathrm{CQ}$, the increased LC3BII levels (LC3B-II/ $\beta$-actin, LC3B-II/LC3B-I, or GFP-LC3 dots) and decreased p62 levels (p62/ $\beta$-actin) were observed in pCMV-myc-PIASy-transfected cells compared with control cells (Fig. 6a, c), whereas the opposite changes of LC3B-II and p62 levels were observed in shRNA-PIASy-transfected cells (Fig. 6b, c). In the presence of $C Q$, the overexpression or silencing expression of PIASy could enhance or decrease the level of LC3B-II, respectively, which was accumulated by $C Q$ inhibition of degradation in lysosomes (Fig. 6a-c); however, the p62 levels were not affected by overexpression or silencing expression of PIASy (Fig. 6a, b), also due to the inhibitory effect of CQ on lysosomes. These results indicated that PIASy itself could activate autophagy in Huh7 cells.

\section{PIASy promotes HCV replication via activation of autophagy in Huh7 cells}

Next, we investigated whether PIASy-induced autophagy contributes to HCV replication in Huh7 cells in the 


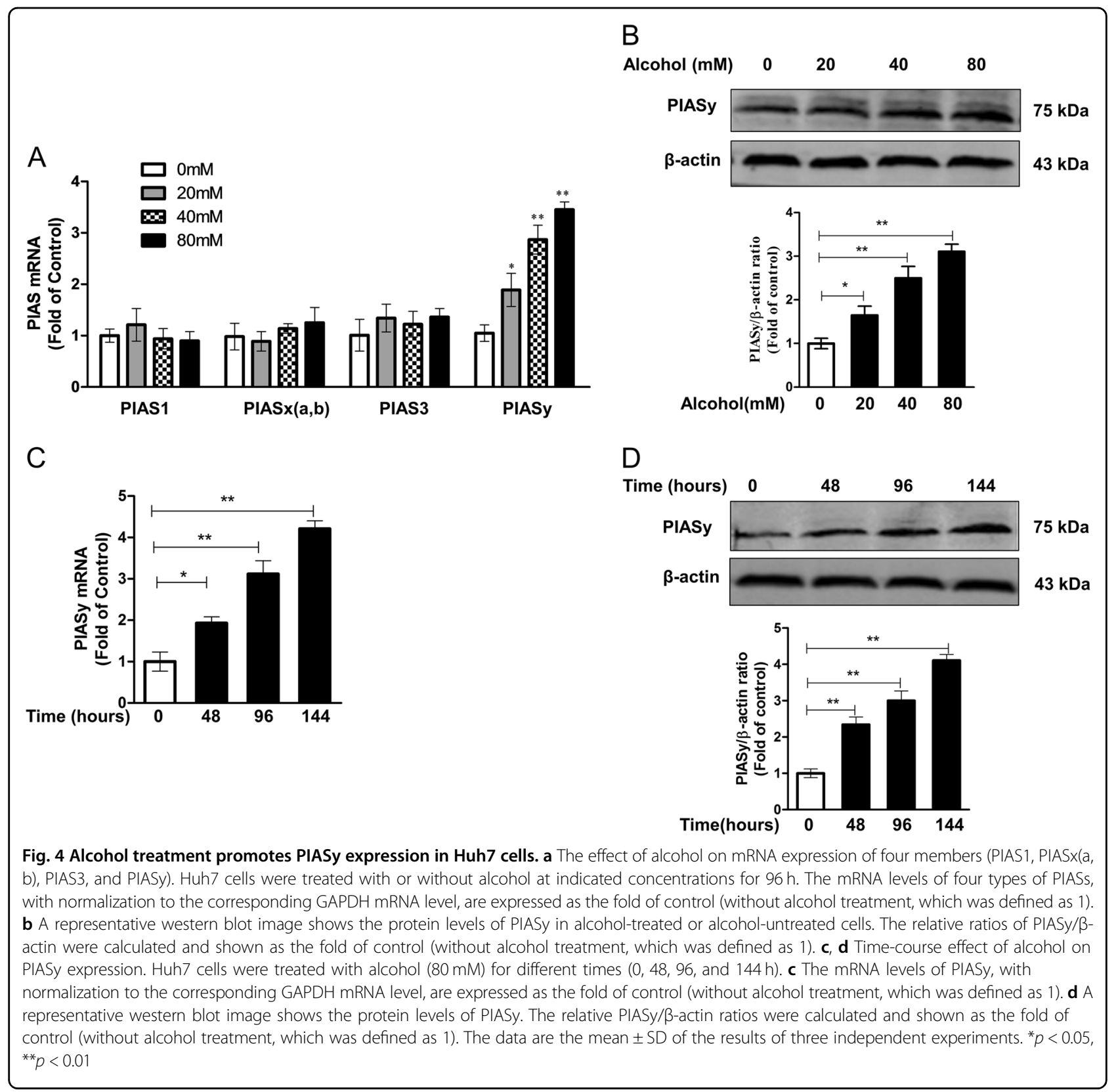

presence or absence of 3-methyladenine (3-MA), an inhibitor in the early stage of autophagy that inhibits conversion of LC3-I into LC3-II. As shown in Fig. 7, 3-MA treatment efficiently led to decreased levels of LC3B-II and suppression of autophagy (Fig. 7a, b). Correspondingly, $\mathrm{HCV}$ core protein levels were also downregulated by 3-MA treatment (Fig. 7a, b), indicating that inhibition of autophagy could suppress HCV replication. In the absence of 3-MA, overexpression of PIASy greatly promoted $\mathrm{HCV}$ replication along with induction of autophagy (Fig. 7a), whereas the silencing expression of PIASy weakened HCV replication along with lessactivated autophagy observed (Fig. 7b). However, in the presence of 3-MA, since the cellular autophagic flux was inhibited, the enhancing effect of PIASy overexpression or the inhibitory effect of silencing expression on $\mathrm{HCV}$ replication were not observed as those in the absence of 3MA (Fig. 7a, b). These results indicated that PIASy promotes HCV replication via activation of autophagy.

\section{PIASy induces autophagy through inducing the} accumulation of SUMO1-conjugated proteins

PIASy belongs to a small ubiquitin-like modifier (SUMO) E3 ligase ${ }^{33}$, and SUMO proteins could modify several components of autophagy to regulate cellular autophagy $^{34,35}$. To assess whether PIASy induces 


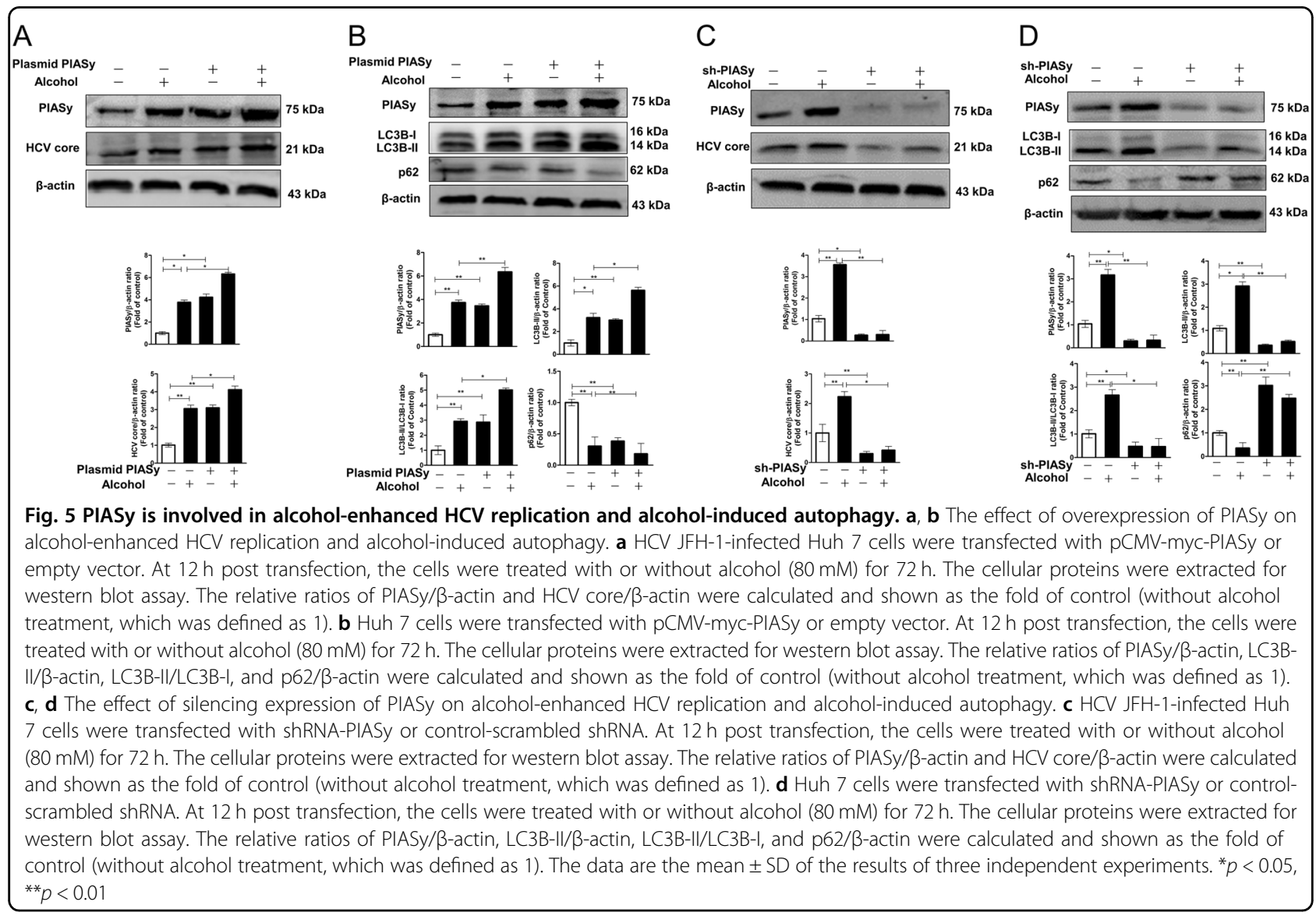

autophagy through the SUMO pathway, we investigate the effect of PIASy on SUMO expression via overexpression or silencing expression of PIASy in Huh7 cells. As shown in Fig. 8, overexpression of PIASy stimulated the expression of SUMO1-conjugated proteins, however, it had little effect on SUMO2/3-conjugated proteins (Fig. 8a). On the contrary, silencing PIASy expression led to decreased expression of SUMO1-conjugated proteins (Fig. 8b), and had little effect on SUMO2/3-conjugated proteins. Since SUMO1- conjugated proteins have a close connection with autophagy, we examined whether the essential and important autophagy-related genes (ATG) were affected by PIASy expression along with the levels of SUMO1-conjugated proteins. Overexpression of PIASy led to increased levels of ATG7 and ATG5-ATG12, but had little effect on ATG3 and Beclin-1 expression (Fig. 8a). Silencing expression of PIASy resulted in decreased levels of ATG7 and ATG5-ATG12, and had little effect on ATG3 and Beclin-1 expression (Fig. 8b).

\section{Discussion}

In the liver, autophagy is an essential mechanism that maintains the balance of energy and nutrients, cellular maintenance, and hepatic function ${ }^{36}$, and it protects the liver from various injuries, including alcohol-induced oxidative stress and accumulation of lipid droplets ${ }^{24,37}$. So far, a number of studies have investigated the effects of alcohol exposure on hepatic autophagy, and most of them indicated that alcohol activates hepatic autophagy ${ }^{24-28}$, but few studies reported that alcohol consumption led to impairment of liver autophagy ${ }^{29}$. Consistent with the results of most of previous studies, we observed that alcohol treatment induces cellular autophagy in Huh7 cells (Figs. 2, 3). Many studies have also shown that autophagy response is induced after HCV infection and HCV hijacks the autophagy machinery to complete infection and replication ${ }^{14,15}$. Therefore, we considered that alcohol-induced autophagy may play a role in the enhancement of HCV replication in liver cells. However, it is hard to illustrate this issue because both alcohol and $\mathrm{HCV}$ infection could induce autophagy in liver cells. Previous studies indicated that alcohol-induced oxidative stress or ER (endoplasmic reticulum) stress, both of which are known to be linked to autophagy activation ${ }^{38}$, are also involved in $\mathrm{HCV}$-induced autophagy ${ }^{39,40}$. Thus, we expected that we could find some new intermediate host factors that are involved in alcohol-induced autophagy but do not derive from HCV infection, to explore the role of alcohol-induced autophagy in HCV replication and the mechanism(s) involved. 


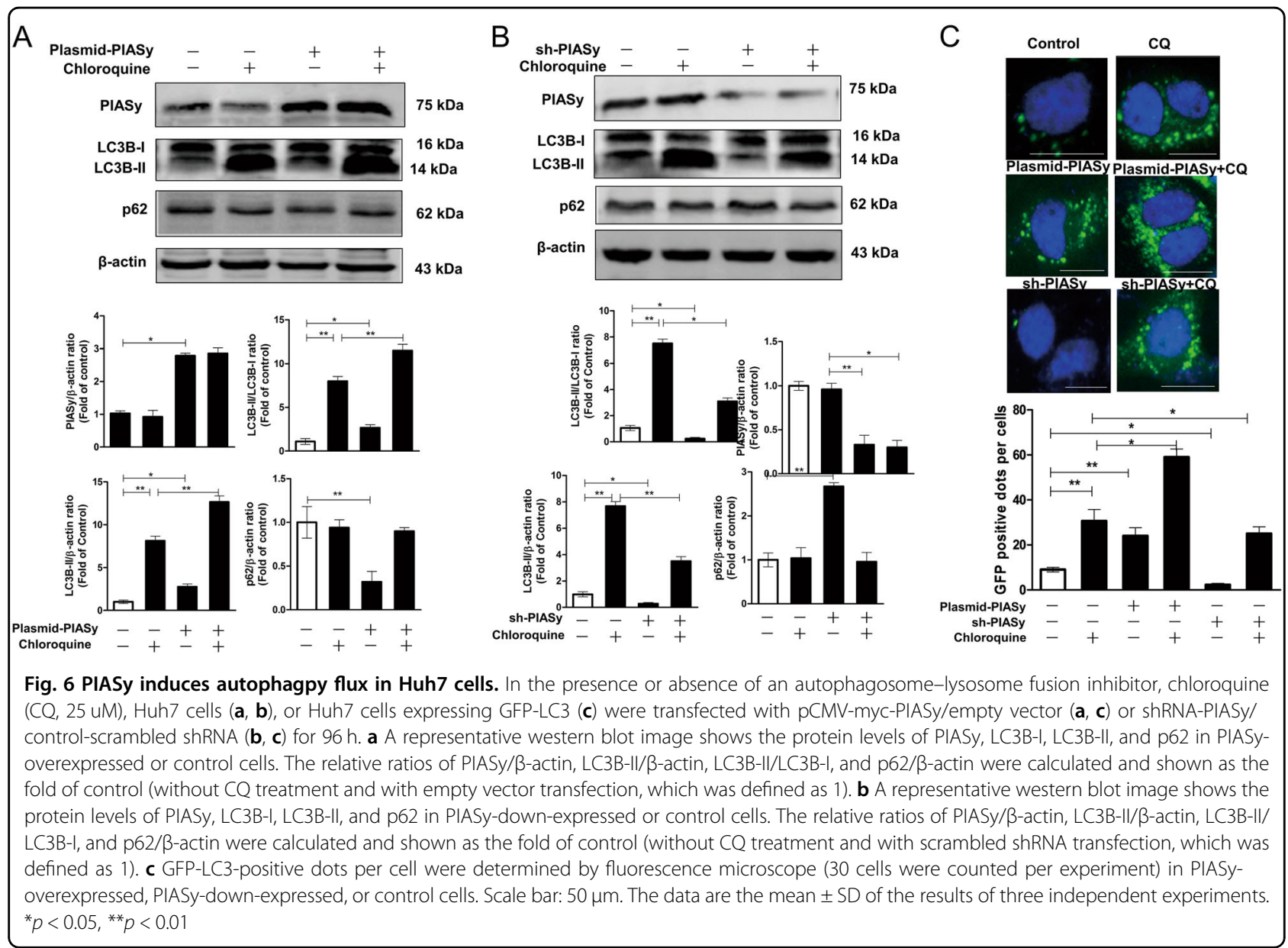

Our preliminary data indicated that alcohol treatment could induce PIASy expression in Huh7 cells, which was confirmed in the present study (Fig. 4). PIASy is a member of PIAS protein family ${ }^{41}$. PIAS represents a family of proteins originally identified as an interaction partner with cytokine-induced STAT, which are negative regulators of innate immunity by repression of key regulators in the interferon (IFN) pathway ${ }^{41-43}$. In addition, PIAS is also known as SUMO E3 ligases ${ }^{33}$, which can serve as a scaffold that brings together SUMO-charged E2 and the substrate, thereby promoting the efficiency and specificity of the sumoylation process. SUMO (small ubiquitin-like modifier protein) is a kind of key posttranslational modification that critically regulates several important cellular functions, including transcription, cell division, protein stability and translocation, signal transduction, protein-protein interactions, and chromatin segregation $^{44,45}$. Recently, several studies have revealed that SUMO proteins play a role in the autophagy pathway. SUMO proteins could modify several components of autophagy to regulate cellular autophagy ${ }^{34,35}$, and SUMO protein itself may undergo autophagosomal degradation ${ }^{46}$. SUMO1 promotes amyloid- $\beta$ production via the modulation of autophagy in Alzheimer's disease ${ }^{47}$. Increased UBC9-mediated SUMOylation is sufficient to induce relatively high levels of autophagy to protect the heart against a proteotoxic environment in cardiac disease $^{48}$. Autophagy induced by elevated levels of SUMO1 impairs synaptic transmission, significantly reducing dendritic spine density and causing memory loss ${ }^{49}$. One recent study reported that PIASy-mediated Tip60 sumoylation regulates p53-induced autophagy ${ }^{50}$. These suggestive data and our preliminary results prompted us to explore the possible linkage between PIASy, SUMO, and autophagy in the process of alcoholpromoting $\mathrm{HCV}$ replication.

We examined whether alcohol-induced PIASy plays a role in cellular autophagy and HCV replication. Silencing expression of alcohol-induced PIASy could decrease the level of autophagy and weaken HCV replication (Fig. 5). More importantly, in the absence of alcohol treatment, PIASy itself possesses the ability to induce autophagy (Figs. 5, 6, and 7). These data provide direct evidence that PIASy is involved in alcohol-induced autophagy and alcohol-enhanced HCV replication. However, when the cellular autophagy was inhibited by the early-stage 


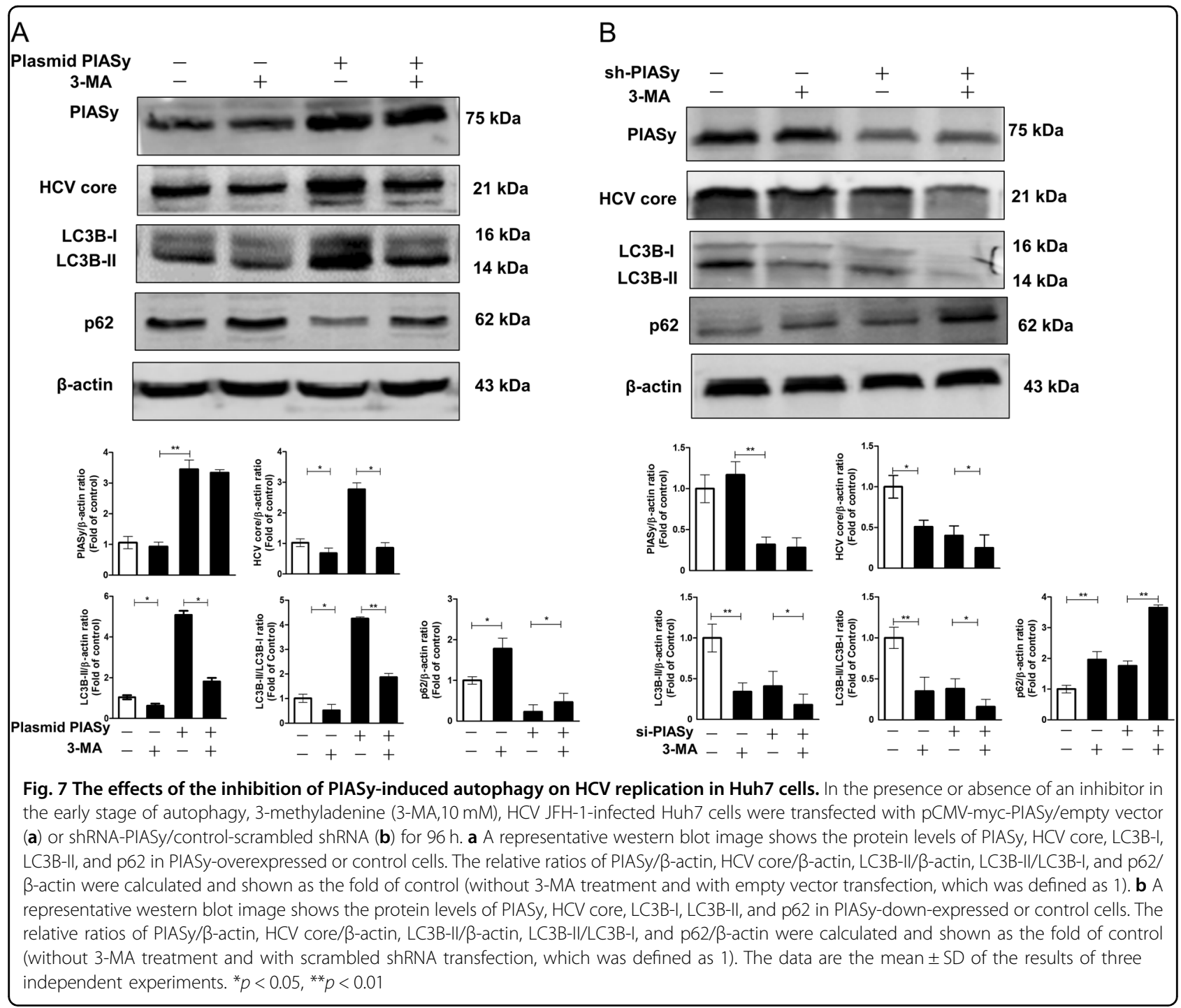

inhibitor 3-MA, the enhancement effect of HCV replication by PIASy was not completely eliminated (Fig. 7a), whereas PIASy-induced autophagy was almost inhibited, indicating that PIASy may promote $\mathrm{HCV}$ replication via other mechanisms. Not surprisingly, PIASy is also a negative regulator of innate immunity by repression of key regulators in the interferon (IFN) pathway ${ }^{41}$, which is always activated by $\mathrm{HCV}$ infection to limit the viral replication $^{51,52}$. In addition, autophagy factors such as ATG5 could directly impact the innate immune pathway, which could negatively regulate type-I IFN pathway and pathogen-associated molecular pattern (PAMP)-mediated cytoplasmic retinoic acid-inducible gene I (RIG-I) pathway $^{15}$, and thus facilitates HCV replication. Therefore, we speculated that the inhibition of the IFN pathway by PIASy, to some extent, compensates the decreased inhibitory effect of ATG5 on the IFN pathway and still enhances $\mathrm{HCV}$ replication under the condition of 3-MA inhibition of autophagy. Nevertheless, because the present study focused on the relationship between PIASy and autophagy, the involvement of the IFN pathway needs further study to illuminate.

In this study, we also observed that PIASy could selectively activate SUMO1 pathway, but not SUMO2/3 pathway (Fig. 8). Overexpression or silencing expression of PIASy could increase or decrease the accumulation of proteins modified by SUMO1, respectively, which is consistent with the previous study ${ }^{53}$. This SUMOylation was also accompanied by increased autophagy formation: increased accumulation of LC3B-II (Fig. 6c) and the formation of GFP-LC3-positive dots (Fig. 6), and the decreased level of p62 expression, which is consistent with some previous studies ${ }^{47,50}$ and implies that increased SUMOylation by PIASy may be a regulator to regulate autophagic flux. In addition, several important autophagy factors, including autophagy-related gene 7 (ATG7) and 


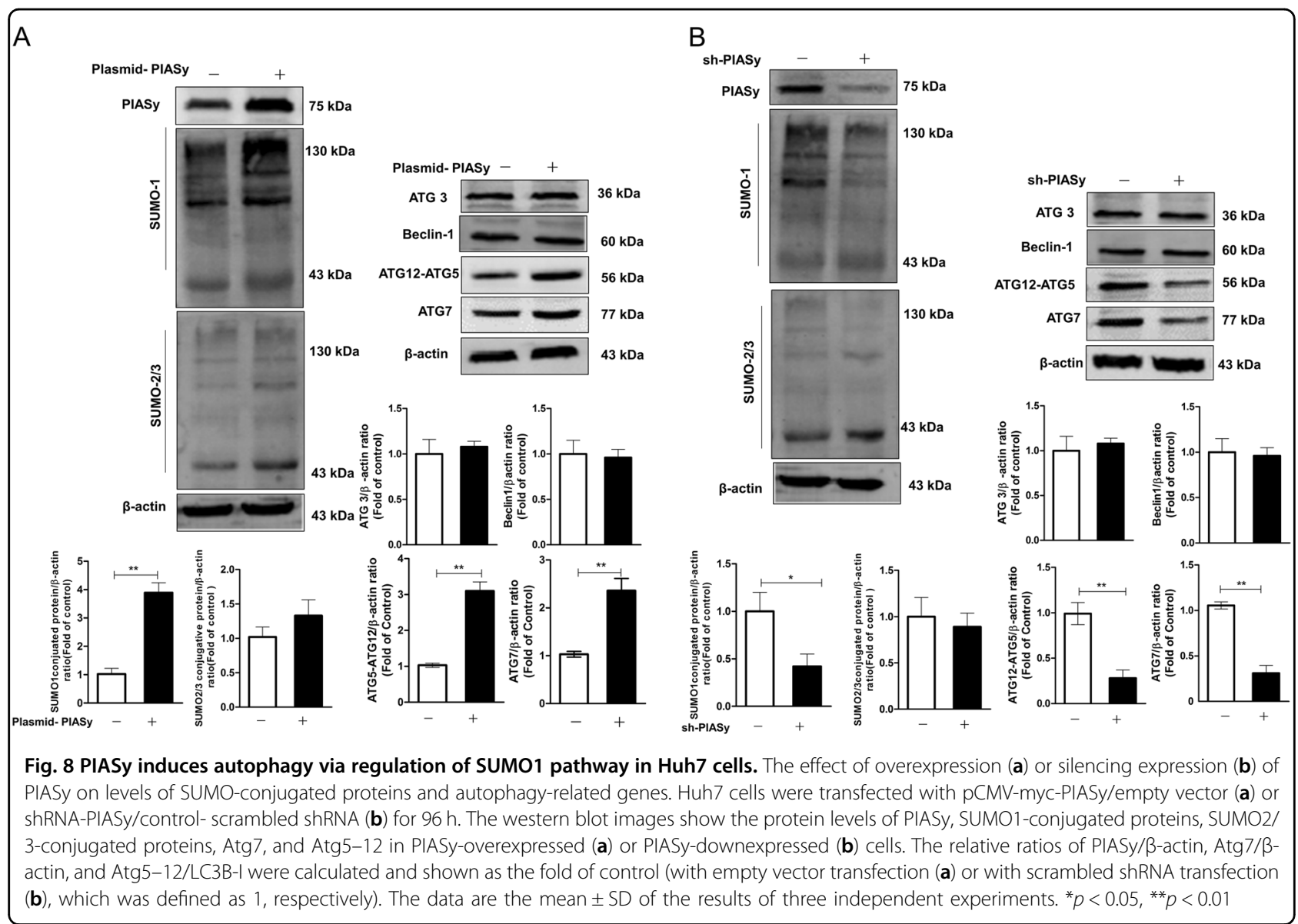

ATG5-ATG12 were significantly induced along with PIASy promoting SUMO1-mediated protein conjugation (Fig. 8). ATG7 and ATG12-ATG5 are key regulators of the autophagic process and play an important role in autophagosome formation. ATG7 plays a role in the early complex of autophagy and the formation of autophagic vacuoles through combining LC3 protein ${ }^{22}$. ATG5 forms a conjugate with ATG12 and 16L1 (ATG16), but the monomeric forms of these two proteins have been shown to be nearly undetectable under normal conditions ${ }^{54}$. The ATG5-ATG12/16L1 complex acts as an E3 enzyme that can promote the formation of autophagic vacuoles and promotes LC3 to be present in autophagic vacuoles ${ }^{55,56}$. Furthermore, direct interaction is transiently linked between ATG5 and HCV NS5B (RNA-dependent RNA polymerase) protein at a very early stage of $\mathrm{HCV}$ infection to facilitate the onset of viral replication ${ }^{17}$. In addition, the ATG5-ATG12 conjugate negatively regulates the type-I IFN production pathway by direct association with the RIG-I and IFN-beta promoter stimulator 1 (IPS-1) through the caspase recruitment domains ${ }^{57}$. And, HCVexploited ATG5 negatively affects cellular immune function-type-I IFN pathway and PAMP-mediated RIG-I signaling ${ }^{15}$.
Taken together, in this study, we presented evidence to show that alcohol promotes HCV replication through activation of autophagy in Huh7 cells. The alcoholactivated autophagy partly attributes to its induction of PIASy expression and thus facilities HCV replication. Our findings provide a novel mechanism for the action of alcohol-promoting $\mathrm{HCV}$ replication in the context of cellular autophagy, which may provide some clues for novel therapeutic strategies to improve anti-HCV effects among alcohol-abused HCV-infected individuals.

\section{Acknowledgements}

This work was supported by the National Natural Science Foundation of China (NO. 81460305, NO. 31360033, and NO. 81360259), Guangxi University "100Talent" Program, and Guangxi university innovation team and outstanding scholars program (NO. Gui Jiao Ren 2014[7]).

\section{Author details}

${ }^{1}$ Guangxi Key Laboratory of AIDS Prevention and Treatment \& Guangxi Universities Key Laboratory of Prevention and Control of Highly Prevalent Disease, School of Public Health, Guangxi Medical University, Nanning 530021 Guangxi, China. ${ }^{2}$ Geriatrics Digestion Department of Internal Medicine, The First Affiliated Hospital of Guangxi Medical University, Nanning 530021 Guangxi, China. ${ }^{3}$ Guangxi Collaborative Innovation Center for Biomedicine, Life Sciences Institute, Guangxi Medical University, Nanning 530021 Guangxi, China. ${ }^{4}$ Department of Pathology and Laboratory Medicine, Temple University School of Medicine, Philadelphia, PA 19140, USA 


\section{Conflict of interest}

The authors declare that they have no conflict of interest.

\section{Publisher's note}

Springer Nature remains neutral with regard to jurisdictional claims in published maps and institutional affiliations.

Supplementary Information accompanies this paper at (https://doi.org/ 10.1038/s41419-018-0845-x).

Received: 4 April 2018 Revised: 28 June 2018 Accepted: 2 July 2018 Published online: 05 September 2018

\section{References}

1. Bhattacharya, R. \& Shuhart, M. C. Hepatitis C and alcohol: interactions, outcomes, and implications. J. Clin. Gastroenterol. 36, 242-252 (2003).

2. McCartney, E. M. \& Beard, M. R. Impact of alcohol on hepatitis C virus replication and interferon signaling. World J. Gastroenterol. 16, 1337-1343 (2010).

3. Polaris Observatory HCV Collaborators. Global prevalence and genotype distribution of epatitis C virus infection in 2015: a modelling study. Lancet Gastroenterol. Hepatol. 2, 161-176 (2017).

4. Maasoumy, B. \& Wedemeyer, H. Natural history of acute and chronic hepatitis C. Best Pract. Res. Clin. Gastroenterol. 26, 401-412 (2012).

5. Gao, B. \& Bataller, R. Alcoholic liver disease: pathogenesis and new therapeutic targets. Gastroenterology 141, 1572-1585 (2011).

6. Anand, B. S. et al. Alcohol use and treatment of hepatitis $C$ virus: results of a national multicenter study. Gastroenterology 130, 1607-1616 (2006).

7. Costentin, C. E. et al. Management of hepatitis $C$ virus infection in heavy drinkers. Alcohol. Alcohol. 48, 337-342 (2013).

8. McCartney, E. M. et al. Alcohol metabolism increases the replication of hepatitis $C$ virus and attenuates the antiviral action of interferon. J. Infect. Dis. 198, 1766-1775 (2008).

9. Ye, L. et al. Alcohol impairs interferon signaling and enhances full cycle hepatitis C virus JFH-1 infection of human hepatocytes. Drug Alcohol. Depend. 112, 107-116 (2010).

10. Choi, J. Oxidative stress, endogenous antioxidants, alcohol, and hepatitis C: pathogenic interactions and therapeutic considerations. Free Radic. Biol. Med. 52, 1135-1150 (2012).

11. Mueller, S., Millonig, G. \& Seitz, H. K. Alcoholic liver disease and hepatitis C: a frequently underestimated combination. World J. Gastroenterol. 15, 3462-3471 (2009).

12. Vieira-Castro, A. C. M. \& Oliveira, L. C. M. Impact of alcohol consumption among patients in hepatitis C virus treatment. Arq. Gastroenterol. 54, 232-237 (2017).

13. Kroemer, G., Marino, G. \& Levine, B. Autophagy and the integrated stress response. Mol. Cell 40, 280-293 (2010).

14. Rautou, P. E. et al. Changes in autophagic response in patients with chronic hepatitis C virus infection. Am. J. Pathol. 178, 2708-2715 (2011).

15. Ke, P. Y. \& Chen, S. S. Activation of the unfolded protein response and autophagy after hepatitis $C$ virus infection suppresses innate antiviral immunity in vitro. J. Clin. Invest. 121, 37-56 (2011).

16. Dreux, M., Gastaminza, P., Wieland, S. F. \& Chisari, F. V. The autophagy machinery is required to initiate hepatitis $C$ virus replication. Proc. Natl Acad. Sci. USA 106, 14046-14051 (2009).

17. Guevin, C. et al. Autophagy protein ATG5 interacts transiently with the hepatitis C virus RNA polymerase (NS5B) early during infection. Virology 405, 1-7 (2010).

18. Tanida, I. et al. Knockdown of autophagy-related gene decreases the production of infectious hepatitis C virus particles. Autophagy 5, 937-945 (2009).

19. Fahmy, A. M. \& Labonte, P. The autophagy elongation complex (ATG5-12/ 16L1) positively regulates HCV replication and is required for wild-type membranous web formation. Sci. Rep. 7, 40351 (2017).

20. Sir, D. et al. Replication of hepatitis C virus RNA on autophagosomal membranes. J. Biol. Chem. 287, 18036-18043 (2012).

21. Wang, L., Tian, Y. \& Ou, J. H. HCV induces the expression of Rubicon and UVRAG to temporally regulate the maturation of autophagosomes and viral replication. PLoS Pathog. 11, e1004764 (2015).
22. Shrivastava, S., Raychoudhuri, A., Steele, R., Ray, R. \& Ray, R. B. Knockdown of autophagy enhances the innate immune response in hepatitis $C$ virusinfected hepatocytes. Hepatology 53, 406-414 (2011).

23. Thomes, P. G., Trambly, C. S., Fox, H. S., Tuma, D. J. \& Donohue, T. M. Jr. Acute and chronic ethanol administration differentially modulate hepatic autophagy and transcription factor EB. Alcohol. Clin. Exp. Res. 39, 2354-2363 (2015).

24. Ding, W. X. et al. Autophagy reduces acute ethanol-induced hepatotoxicity and steatosis in mice. Gastroenterology 139, 1740-1752 (2010).

25. Thomes, P. G. et al. Proteasome activity and autophagosome content in liver are reciprocally regulated by ethanol treatment. Biochem. Biophys. Res. Commun. 417, 262-267 (2012)

26. Sid, B., Verrax, J. \& Calderon, P. B. Role of AMPK activation in oxidative cell damage: Implications for alcohol-induced liver disease. Biochem. Pharmacol. 86, 200-209 (2013).

27. Ni, H. M., Du, K., You, M. \& Ding, W. X. Critical role of FoxO3a in alcohol-induced autophagy and hepatotoxicity. Am. J. Pathol. 183, 1815-1825 (2013).

28. Lin, C. W. et al. Pharmacological promotion of autophagy alleviates steatosis and injury in alcoholic and non-alcoholic fatty liver conditions in mice. J. Hepatol. 58, 993-999 (2013).

29. Wu, D., Wang, X., Zhou, R., Yang, L. \& Cederbaum, A. I. Alcohol steatosis and cytotoxicity: the role of cytochrome P4502E1 and autophagy. Free Radic. Biol. Med. 53, 1346-1357 (2012).

30. Wakita, T. et al. Production of infectious hepatitis $C$ virus in tissue culture from a cloned viral genome. Nat. Med. 11, 791-796 (2005).

31. Zhang, $T$. et al. Alcohol potentiates hepatitis $C$ virus replicon expression. Hepatology 38, 57-65 (2003).

32. Zhuang, Y., Li, Y., Li, X., Xie, Q. \& Wu, M. Atg7 knockdown augments concanavalin A-induced acute hepatitis through an ROS-mediated p38/MAPK pathway. PLOS ONE 11, e0149754 (2016).

33. Palvimo, J. J. PIAS proteins as regulators of small ubiquitin-related modifier (SUMO) modifications and transcription. Biochem. Soc. Trans. 35, 1405-1408 (2007).

34. Yang, Y. et al. Acetylated hsp70 and KAP1-mediated Vps34 SUMOylation is required for autophagosome creation in autophagy. Proc. Natl Acad. Sci. USA 110, 6841-6846 (2013).

35. Cajee, U. F., Hull, R. \& Ntwasa, M. Modification by ubiquitin-like proteins: significance in apoptosis and autophagy pathways. Int. J. Mol. Sci. 13 11804-11831 (2012).

36. Yin, X. M., Ding, W. X. \& Gao, W. Autophagy in the liver. Hepatology 47, 1773-1785 (2008)

37. Wu, D., Wang, X., Zhou, R. \& Cederbaum, A. CYP2E1 enhances ethanol-induced lipid accumulation but impairs autophagy in HepG2 E47 cells. Biochem. Biophys. Res. Commun. 402, 116-122 (2010).

38. Ding, W. X. \& Yin, X. M. Sorting, recognition and activation of the misfolded protein degradation pathways through macroautophagy and the proteasome. Autophagy 4, 141-150 (2008).

39. Medvedev, R. et al. HCV-induced oxidative stress by inhibition of Nrf2 triggers autophagy and favors release of viral particles. Free Radic. Biol. Med. 110, 300-315 (2017)

40. Dash, S. et al. Hepatitis C virus infection induces autophagy as a prosurvival mechanism to alleviate hepatic ER-stress response. Viruses 8, E150 (2016).

41. Liu, B., Gross, M., ten, Hoeve, J. \& Shuai, K. A transcriptional corepressor of Stat1 with an essential LXXLL signature motif. Proc. Natl Acad. Sci. USA 98, 3203-3207 (2001)

42. Liu, B. et al. PIAS1 selectively inhibits interferon-inducible genes and is important in innate immunity. Nat. Immunol. 5, 891-898 (2004).

43. Arora, T. et al. PIASx is a transcriptional co-repressor of signal transducer and activator of transcription 4. J. Biol. Chem. 278, 21327-21330 (2003).

44. Geiss-Friedlander, R. \& Melchior, F. Concepts in sumoylation: a decade on. Nat. Rev. Mol. Cell Biol. 8, 947-956 (2007).

45. Cubenas-Potts, C. \& Matunis, M. J. SUMO: a multifaceted modifier of chromatin structure and function. Dev. Cell. 24, 1-12 (2013).

46. Mattoscio, D. et al. Autophagy regulates UBC9 levels during viral-mediated tumorigenesis. PLoS Pathog. 13, e1006262 (2017).

47. Cho, S. J. et al. SUMO1 promotes Abeta production via the modulation of autophagy. Autophagy 11, 100-112 (2015).

48. Gupta, M. K. \& Robbins, J. Making the connections: autophagy and posttranslational modifications in cardiomyocytes. Autophagy 12, 2252-2253 (2016).

49. Matsuzaki, S. et al. SUMO1 affects synaptic function, spine density and memory. Sci. Rep. 5, 10730 (2015). 
50. Naidu, S. R., Lakhter, A. J. \& Androphy, E. J. PIASy-mediated Tip60 sumoylation regulates p53-induced autophagy. Cell Cycle 11, 2717-2728 (2012).

51. Heim, M. H. \& Thimme, R. Innate and adaptive immune responses in HCV infections. J. Hepatol. 61, S14-S25 (2014).

52. Kawai, T. \& Akira, S. The roles of TLRS, RLRs and NLRs in pathogen recognition. Int. Immunol. 21, 317-337 (2009).

53. Cui, W. et al. SUMOylation and ubiquitylation circuitry controls pregnane $X$ receptor biology in hepatocytes. Drug Metab. Dispos. 43, 1316-1325 (2015).
54. Mizushima, N. et al. Mouse Apg16L, a novel WD-repeat protein, targets to the autophagic isolation membrane with the Apg12-Apg5 conjugate. J. Cell Sci. 116, 1679-1688 (2003).

55. Hanada, T. et al. The Atg12-Atg5 conjugate has a novel E3-like activity for protein lipidation in autophagy. J. Biol. Chem. 282, 37298-37302 (2007).

56. Walczak, M. \& Martens, S. Dissecting the role of the Atg12-Atg5-Atg16 complex during autophagosome formation. Autophagy 9, 424-425 (2013).

57. Jounai et al. The Atg5 Atg12 conjugate associates with innate antiviral immune responses. Proc. Natl Acad. Sci. USA 104, 14050-14055 (2007). 